Revista Brasileira de Agricultura Irrigada v.11, nº.7, p. 1966 - 1974, 2017

ISSN 1982-7679 (On-line)

Fortaleza, CE, INOVAGRI - http://www.inovagri.org.br

DOI: $10.7127 /$ rbai.v11n700663

Protocolo 663.17 - 17/04/2017 Aprovado em 08/09/2017

\title{
EFICIÊNCIA DO USO DA ÁGUA DE IRRIGAÇÃO NO CULTIVO DE BANANA (Musa sp. L.)
}

\author{
Antônio Erivando Bezerra ${ }^{1}$, Carlos Wagner Oliveira² ${ }^{2}$ João Miguel de Morais Neto ${ }^{3}$, Toshik \\ Iarley da Silva ${ }^{4}$, Ana Célia Maia Meireles ${ }^{5}$, Hernandes Rufino dos Santos ${ }^{6}$
}

\begin{abstract}
RESUMO
A banana (Musa sp.) é um dos frutos mais consumidos mundialmente, no entanto, requer elevada demanda hídrica para atingir o seu potencial produtivo. Para que se possa obter equilíbrio entre consumo e eficiência do uso da água, torna-se necessário a implementação do gerenciamento integrado dos recursos hídricos. Com vista nisso, o objetivo desse trabalho foi utilizar dados de evapotranspiração como parâmetro para determinar a eficiência da irrigação no cultivo de banana nos municípios de Barbalha e Missão Velha, CE, através de sensoriamento remoto. O cultivo demostrou-se estável e consolidado há mais de dois anos, apresentando normalidade na taxa evaporativa entre 75 e 100\%, indicando que o nível de estresse hídrico ao qual a cultura esteve submetida foi baixo. Haja vista o manejo empregado na irrigação e a taxa de evapotranspiração obtida encontrar-se próxima de 100\%, percebe-se que o cultivo da empresa Sítio Barreiras proporciona melhor eficiência no uso da água, portanto mantendo o nível de produtividade em torno de 25-30 t ha-1.
\end{abstract}

Palavras-chave: monitoramento, demanda hídrica, irrigação.

\section{EFFICIENCY OF THE USE OF THE IRRIGATION WATER IN THE BANANA CULTIVE (Musa sp. L.)}

\begin{abstract}
Banana (Musa sp.) is one of the most consumed fruits worldwide, however, it requires elevated hydric demand to reach it is productive potential. For that enlace to achieve the balance between consumption and efficiency of water use, it is necessary the implementation of the management integrated of the water resources. In view of this, the aim of this work was to utilize evapotranspiration's data as parameter to determine the efficiency of irrigation in

\footnotetext{
${ }^{1}$ Mestrando em Engenharia Agrícola, UFCG, Campina Grande-PB, e-mail: aerivando86@gmail.com

${ }^{2} \mathrm{PhD}$ em Biosystems Engineering, Professor da UFCA, Crato-CE, e-mail: carlos.oliveira@ufca.edu.br

${ }^{3}$ Doutor em Recursos Naturais, Professor da UFCG, Campina Grande-PB, e-mail: moraes@deag.ufcg.edu.br

${ }^{4}$ Mestrando em Agronomia, UFPB, Areia-PB, e-mail: iarley.toshik@gmail.com

${ }^{5}$ Doutora em Engenharia Civil (Recursos Hídricos), Professora da UFCA, Crato-CE, e-mail: ana.meireles@ufca.edu.br

${ }^{6}$ Doutor em Fitotecnia, Crato-CE, e-mail: hernandes.rufino@ufca.edu.br
} 
banana cultivation in the cities of Barbalha and Missão Velha, CE, through remote sensing. The cultivation was shown to be stable and consolidated for more than two years, showing normality in the evaporative rate between 75 and 100\%, indicating that the level of hydric stress to which the crop was submitted was low. In view the management used in irrigation and the evapotranspiration rate obtained is close to $100 \%$, it is perceivered that the cultivation of the company Sítio Barreiras provides better efficiency in the use of water, therefore maintaining the level of productivity around 25-30 t ha-1.

Keywords: monitoring, hydric demand, irrigation.

\section{INTRODUÇÃO}

A bananeira (Musa sp. L.) pertence à família Musaceae, sendo um vegetal herbáceo completo constituído por sistema radicular, caule subterrâneo (rizoma), pseudocaule, folhas, flores e frutos, e em alguns casos, sementes (COELHO et al., 2012). A banana ocupa primeira posição no ranking mundial das frutas, com uma produção de 106,5 milhões de toneladas (FAO, 2016). O Brasil é o quinto produtor mundial desse fruto, com aproximadamente 7,0 milhões de toneladas produzidas, área colhida de 480,8 mil hectares e rendimento médio de 14,2 $\mathrm{t} \mathrm{ha}^{-1}$ (IBGE, 2010). A Região Nordeste é a maior produtora desse fruto, sendo seguida das Regiões Norte, Sudeste, Sul e Centro-Oeste. No entanto, é preciso superar algumas limitações na baixa eficiência na produção, tendo em vista que as localidades onde a bananeira é cultivada, as precipitações são insuficientes para atender às necessidades hídricas, tornando-se necessário o uso de irrigação suplementar, como ocorre no semiárido nordestino (MONTENEGRO et al., 2009).

O censo agropecuário estadual realizado no ano de 2010 revela que o cultivo de banana no Estado do Ceará, ocupa área de aproximadamente 50,5 mil hectares, com produção média de aproximadamente 436,2 mil toneladas e produtividade média de $8,7 \mathrm{t}$ $\mathrm{ha}^{-1}$, sendo esses valores são relativamente baixos em comparação com outros estados produtores da fruta. No polo agrícola irrigado da Região Metropolitana do Cariri, os municípios de Barbalha e Missão Velha são os maiores produtores de banana (ADECE, 2013).
A estimativa do uso consuntivo de água, da sanidade e eficiência produtiva da bananeira ocorre principalmente pela determinação da evapotranspiração da cultura (ETc), sendo a mesma ferramenta indispensável para realização do manejo adequado da irrigação, contribuindo para redução do desperdício de água, favorecendo o desenvolvimento da cultura e elevando os índices de produtividade (COELHO et al., 2012). Para manter o nível de produtividade de cultivos extremamente sensíveis ao déficit hídrico, por exemplo da bananeira, recomenda-se que o nível de estresse hídrico não fique abaixo da faixa compreendida entre 50 e $65 \%$ da taxa de evapotranspiração (SHAPLAND, 2015).

Os modelos clássicos de medição da evapotranspiração fornecem informações pontuais, utilizando o balanço hídrico no solo ou lisímetro, por exemplo. No entanto, a ETc, obtida por esses métodos, apresenta custo e grau de complexidade elevado, uma vez que a taxa de evapotranspiração da cultura é fortemente influenciada pelo tipo de vegetação, manejo agrícola, gestão ambiental e, principalmente, por parâmetros climáticos incluindo radiação solar, vento, temperatura e umidade relativa (ALLEN et al., 2007).

Com o intuito de reduzir custos e melhorar a gestão agrícola, nas últimas décadas foram desenvolvidos vários produtos de sensoriamento remoto, principalmente com utilização de imagens de satélite. Essa tecnologia, fornece dados precisos, de maneira rápida, a baixo custo, com eficiência significativa, podendo ser empregado na obtenção de índices de qualidade sanitária da cultura agrícola, balanço de radiação na superfície, taxa de variabilidade da 
evapotranspiração real, produção de biomassa e eficiência do uso da água, entre outros (SILVA et al., 2013; SOUZA et al., 2014; TEIXEIRA et al., 2014).

A grande vantagem desse método é permitir estimativas em escala regional, contemplando áreas com grande variabilidade de solo, vegetação, precipitação, entre outros. De modo que os resultados possam ser expressos na forma de mapas digitais melhorando o planejamento hidro agrícola (ROMÁN et al., 2010). Com vista nisso, o objetivo desse trabalho foi utilizar dados de evapotranspiração como parâmetro para determinar a eficiência da irrigação no cultivo de banana nos municípios de Barbalha e Missão Velha, CE, através de sensoriamento remoto.

\section{MATERIAL E MÉTODOS}

A pesquisa foi desenvolvida em bananal localizado no distrito de Missão Nova, no sudeste do estado do Ceará, conforme Figura 1. A área está situada no município de Missão Velha, com latitude de $7^{\circ} 19^{\prime}$ sul, longitude de $39^{\circ} 11^{\prime}$ oeste de Greenwich e altitude de 403 metros. Nesse local encontram-se instaladas duas das maiores empresas do ramo de fruticultura irrigada da região, sendo elas o Sítio Barreiras (STB) com área aproximada de 358 hectares (sendo 326,1 hectares cultivados com a variedade Prata Anã e 32,5 hectares da variedade Nanica) e o Sítio Paraiso Verde (STPV) com área aproximada de 460 hectares (sendo 446 hectares cultivados com a variedade Prata Anã e 14 hectares da variedade Nanica).

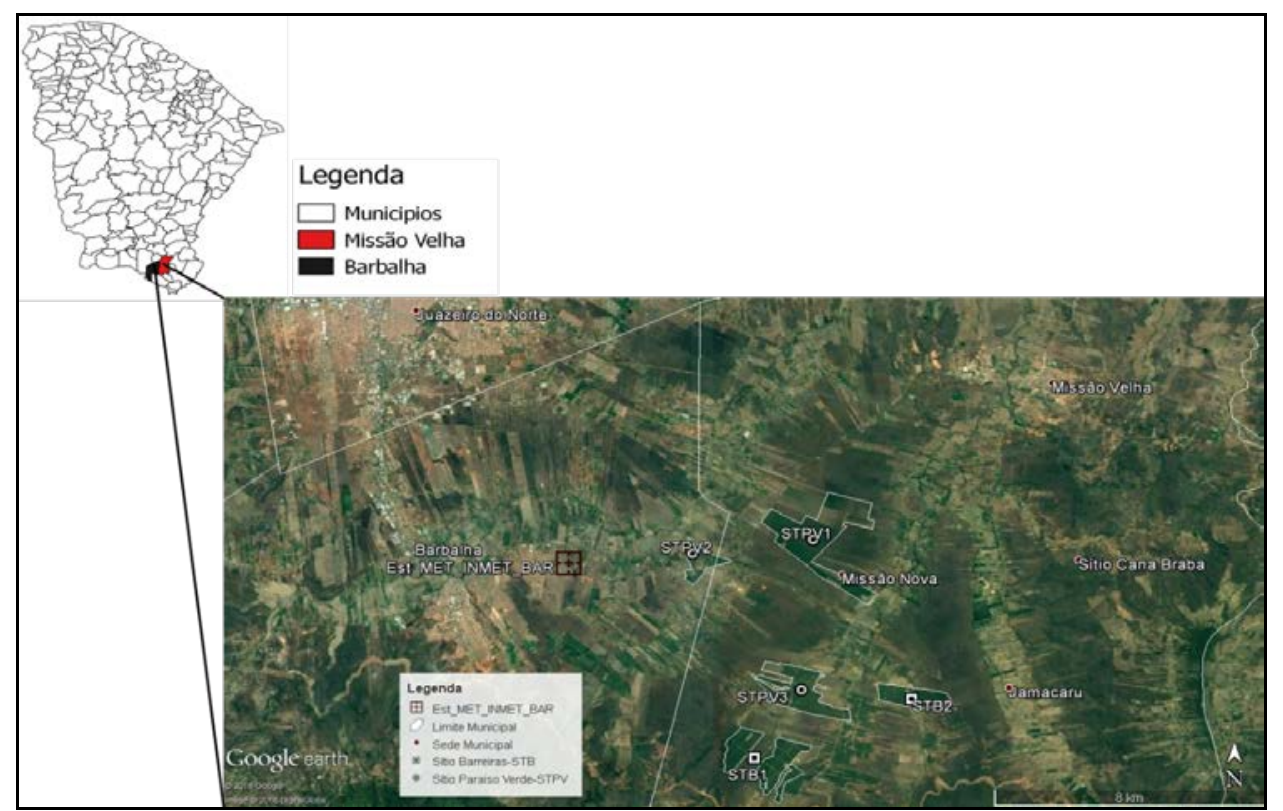

Figura 1. Localização da área em estudo, destacando o cultivo de banana das empresas Sítio Paraiso Verde (STPV) e Sítio Barreiras (STB).

FONTE: Adaptado do Google Earth (2016).

O clima predominante na localidade em estudo é quente e úmido (Aw), com regime de chuvas do tipo tropical austral (w) dividido em duas etapas, sendo a primeira etapa com chuvas concentradas no período de janeiro a abril, e a segunda com seca estacional iniciando em maio e perdurando até dezembro, totalizando 1.075,8 $\mathrm{mm}$ de precipitação média anual (BRANDÃO et al.,
2012). A área apresenta condições edafoclimáticas favoráveis ao desenvolvimento da bananicultura, haja vista que a temperatura média anual situa-se próximo de $24,9{ }^{\circ} \mathrm{C}$, umidade relativa superior a $80 \%$, taxa de insolação de 2.848 horas anual e velocidade média do vento próximo a 1,90 $\mathrm{m} \mathrm{s}^{-1}$, (MEDEIROS et al., 2013). 
O cultivo da banana ocorre principalmente sobre duas manchas de solos específicas, representadas na Figura 2. O ARGISSOLO VERMELHO-PV1 é um solo relativamente plano, profundo, bem drenado, porém necessitando de adição de matéria orgânica para melhorar a fertilidade e aumentar a retenção de umidade, já o NEOSSOLO FLÚVICO-RY3 é caracterizado como sendo um solo de formação sedimentar, profundo, bem drenado e rico em matéria orgânica
(FUNCEME, 2012).

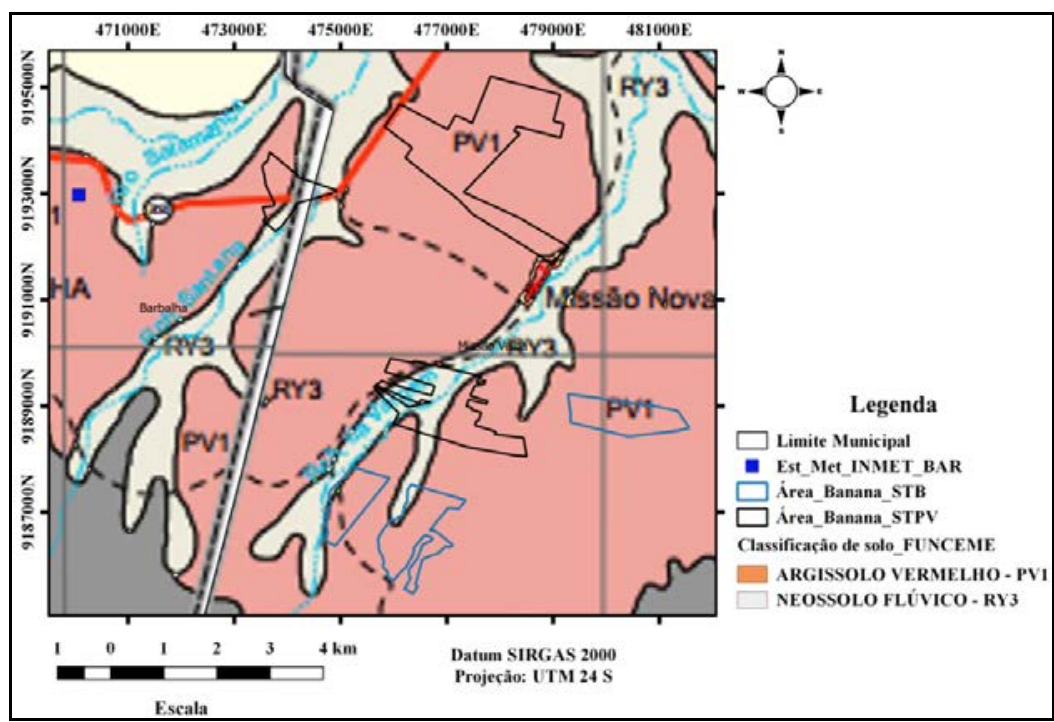

Figura 2. Classificação de solos da área de estudo destacando os tipos de solos onde estão instaladas as empresas Sítio Barreiras (STB) e Sítio Paraiso Verde (STPV).

FONTE: Adaptado de FUNCEME (2012).

De forma resumida o fluxograma esquemático, representado na Figura 3, descreve como foi realizado o compilamento dos dados e o processamento do modelo METRIC, para obtenção dos componentes do balanço de energia e taxa de evapotranspiração da cultura.

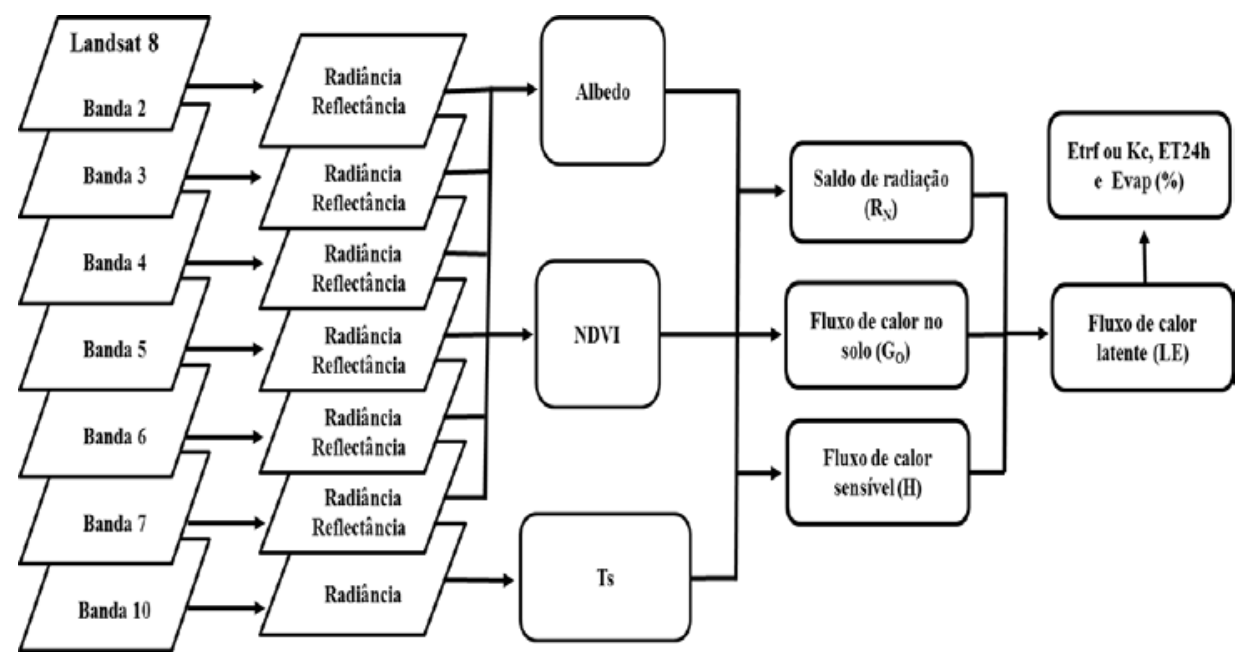

Figura 3. Fluxograma esquemático do processamento dos dados para obtenção do balanço de energia e ET.

O algoritmo METRIC possui várias etapas sequenciais, pois em cada uma delas é feito o cálculo de uma ou mais variáveis necessárias para a etapa seguinte (ALLEN et al., 2005). Este método calcula a taxa evaporativa da cultura através do balanço de energia na superfície, utilizando imagens de satélite e algumas variáveis meteorológicas obtidas em estações meteorológicas automáticas (PCD’s). Tais como: 
temperatura, velocidade do vento, temperatura do ponto de orvalho, evapotranspiração de referência, pressão atmosférica, insolação e precipitação. O processamento dos dados da estação meteorológica, das imagens e do modelo foi realizado no software ArcGis versão 10.2 .

As imagens do satélite LANDSAT 8 (OLI) referente ao caminho 217 e linha 65, foram adquiridas gratuitamente junto à USGS (United States Geological Survey) na plataforma "Earth Explorer", disponível no site http://earthexplorer.usgs.gov/, contemplando as seguintes datas: 03 de março, 22 de maio, 09 de julho, 29 de outubro e 14 de novembro de 2016. A escolha das imagens está condicionada à baixa cobertura de nuvens e a excelente qualidade de processamento, atendendo perfeitamente aos requisitos da pesquisa. Os dados de clima utilizados no processo de validação do modelo e no cálculo da $\mathrm{ET}_{0}$, foram coletados pela estação meteorológica automática de Barbalha, pertencente ao Instituto Nacional de Meteorologia (INMET), localizada nas seguintes coordenadas: Latitude $7^{\circ} 18^{\prime}$ Sul, Longitude $39^{\circ} 16^{\prime}$ Oeste, 409,03 metros de altitude e distante 13 quilômetros das áreas cultivadas com banana.

Para iniciar o processamento das imagens tornou-se necessária a identificação de alguns parâmetros de ajustes como: Ângulo de inclinação solar - E (grau), Cosseno do ângulo zenital - $\operatorname{Cos} \mathrm{Z}\left({ }^{\circ}\right)$, Temperatura do Ar-Ta $\left({ }^{\circ} \mathrm{C}\right)$, Umidade Relativa do Ar - RU (\%), Pressão atmosférica - Po (kPa), Transmitância Atmosférica - ( oc) e distância média entre a Terra e o Sol - Dr (unidade astronômica), para uma referida data no calendário Juliano (DJ), apresentado na Tabela 1 e alguns parâmetros de calibração, apresentado na $\quad$ Tabela 2.

Tabela 1. Variáveis utilizadas para calcular a Reflectância, Transmitância e Albedo.

\begin{tabular}{ccccccccc}
\hline Data & DJ & E & Cos Z & Ta & UR & Po & $\tau_{\text {oc }}$ & D \\
\hline $03 / 03 / 2016$ & 63 & 59,29 & 0,86 & 28,25 & 53,5 & 96,6 & 0,742 & 0,9914 \\
$22 / 05 / 2016$ & 143 & 51,43 & 0,78 & 27,90 & 50,0 & 96,8 & 0,737 & 1,0124 \\
$09 / 07 / 2016$ & 191 & 48,85 & 0,75 & 26,50 & 52,2 & 97,0 & 0,736 & 1,0166 \\
$29 / 10 / 2016$ & 303 & 65,74 & 0,90 & 28,85 & 43,0 & 96,6 & 0,768 & 0,9931 \\
$14 / 11 / 2016$ & 319 & 64,11 & 0,89 & 30,45 & 45,5 & 96,2 & 0,754 & 0,9891 \\
\hline
\end{tabular}

FONTE: Adaptado de Silva et al (2016).

Tabela 2. Coeficientes de calibração para as bandas do satélite Landsat 8 (OLI).

\begin{tabular}{cccccc}
\hline \multirow{2}{*}{ Bandas } & $\begin{array}{c}\text { Coeficientes de } \\
\text { Calibração } \\
\left(\mathrm{w} \mathrm{m}^{-2} \mathrm{st}^{-1} \mu^{-1}\right)\end{array}$ & \multicolumn{2}{c}{$\begin{array}{c}\text { Quantizado e calibrado do } \\
\text { pixel em nível de cinza } \\
(\mathrm{ND})\end{array}$} & $\begin{array}{c}\text { Irradiância Espectral } \\
\text { ESUN_TOA } \\
\left(\mathrm{w} \mathrm{m}^{-2} \mu^{-1}\right)\end{array}$ \\
\cline { 2 - 5 } Lmin & Lmax & Qcalmin & Qcalmax & \\
\hline Banda 2 & $-65,4$ & 791,7 & 1 & 65535 & 2006 \\
Banda 3 & $-60,2$ & 729,5 & 1 & 65535 & 1818 \\
Banda 4 & $-50,8$ & 615,2 & 1 & 65535 & 1553 \\
Banda 5 & $-31,1$ & 376,5 & 1 & 65535 & 1003 \\
Banda 6 & $-7,7$ & 93,6 & 1 & 65535 & 250,9 \\
Banda 10 & 0,1 & 22,0 & 1 & 65535 & 1 \\
Banda 7 & $-2,6$ & 31,1 & 1 & 65535 & 89,59 \\
\hline
\end{tabular}

FONTE: Adaptado de USGS (2016).

As etapas computacionais do algoritmo MECTRIC para a obtenção do fluxo de calor latente (LE) chega ao fim quando o mesmo é colocado em evidência e realiza-se a simples diferença entre o saldo de radiação (Rn), o fluxo de calor no solo $(G)$ e o 
fluxo de calor sensível (H), conforme a equação 1:

$$
\mathrm{LE}=\mathrm{Rn}-\mathrm{G}-\mathrm{H}
$$

Em que:

$\mathrm{Rn}=$ saldo de radiação $\left(\mathrm{Wm}^{-2}\right)$;

$\mathrm{G}=$ fluxo de calor para solo $\left(\mathrm{Wm}^{-2}\right)$;

$\mathrm{H}=$ fluxo de calor sensível $\left(\mathrm{Wm}^{-2}\right)$;
$\mathrm{LE}=$ representa o fluxo de calor latente $\left(\mathrm{Wm}^{-}\right.$ $\left.{ }^{2}\right)$.

A evapotranspiração de referência em 24 horas $\left(\mathrm{ET}_{0} \_24 \mathrm{~h}\right)$ foi obtida pelo método de Penman-Monteith-FAO56, através do software REF-ET (ALLEN, 2002), utilizando os dados coletados pela (PCD's), tendo como referência uma cultura hipotética de altura 0,12 m, um valor fixo de resistência da superfície de $70 \mathrm{~s} \mathrm{~m}^{-1}$ e albedo de 0.23 , conforme a equação 2 :

$$
\mathrm{ET}_{0}=\frac{0,408 \Delta(\mathrm{Rn}-\mathrm{G})+\gamma \frac{900}{\mathrm{~T}+273} \mathrm{U}_{2}\left(\mathrm{e}_{\mathrm{s}}-\mathrm{e}_{\mathrm{a}}\right)}{\Delta+\gamma\left(1+0.34 \mathrm{U}_{2}\right)}
$$

Em que:

$\mathrm{ET}_{0}=$ evapotranspiração da cultura de referência $\left(\mathrm{mm} \mathrm{d}^{-1}\right)$;

$\mathrm{Rn}=$ radiação líquida na superfície da cultura $\left(\mathrm{Mj} \mathrm{m}^{-2} \mathrm{dia}^{-1}\right)$;

$\mathrm{G}=$ fluxo de calor do solo $\left(\mathrm{Mj} \mathrm{m}^{-2} \mathrm{dia}^{-1}\right)$;

$\mathrm{T}=$ temperatura média do ar $\left({ }^{\circ} \mathrm{C}\right)$;

$\mathrm{U}_{2}=$ velocidade do vento medida a $2 \mathrm{~m}$ de altura $\left(\mathrm{m} \mathrm{s}^{-1}\right)$;

$\mathrm{e}_{\mathrm{s}}-\mathrm{e}_{\mathrm{a}}=$ déficit da pressão de vapor (Kpa);

$\Delta=$ variação da declividade da curva da pressão de vapor (Kpa);

$\gamma=$ constante psicométrica $\left(\mathrm{Kpa}^{\circ} \mathrm{C}\right)$;

900 = fator de conversão.

A taxa de evapotranspiração da cultura (Evap\%) correspondente a porcentagem evaporativa decorrente em uma determinada área de cultivo com banana, foi determinada por meio da multiplicação da fração de evapotranspiração pelo o fator correspondente a maior taxa evaporativa, ou seja, $100 \%$ da evapotranspiração da cultura, segundo a equação 3:

$$
\operatorname{Evap}(\%)=\operatorname{ETrf} \mathrm{x} f
$$

Em que:

$\operatorname{Evap}_{(\%)}=$ taxa de evapotranspiração da cultura (\%);

ETrf = fração de evapotranspiração ou Kc;

$\mathrm{f}=$ fator correspondente a máxima evapotranspiração ou $\quad 100 \%$ de evapotranspiração da cultura.

\section{RESULTADOS E DISCUSSÕES}

De acordo com a metodologia descrita, os resultados obtidos demostram que o cultivo de banana da localidade em estudo apresenta taxa evaporativa no intervalo entre $70 \%$ e 90\% durante a maioria dos meses analisados, como representado na Figura 4. Nos meses de março, maio e julho a taxa evaporativa da cultura encontrou-se no intervalo entre 70 a $90 \%$, como mostram as figuras $4 \mathrm{~A}, \mathrm{~B}$ e C. O maior pico da taxa evaporativa ocorreu nos meses de outubro e novembro, como evidencia as figuras $4 \mathrm{D}$ e $\mathrm{E}$, com valores entre $90 \%$ e $100 \%$ em quase totalidade das áreas 
(A)

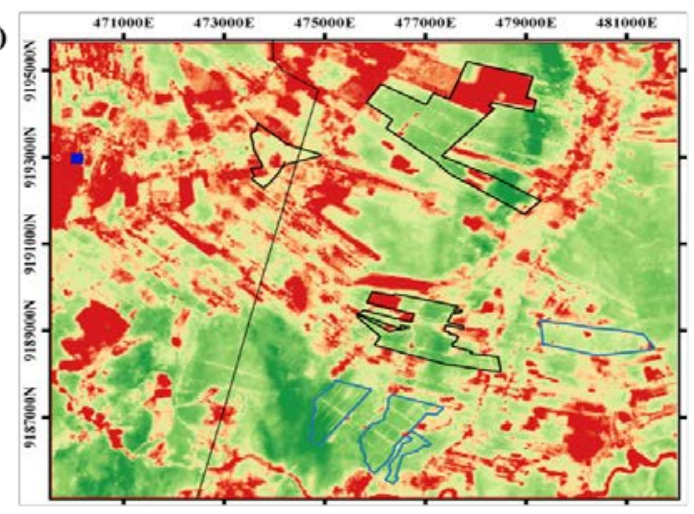

(C)

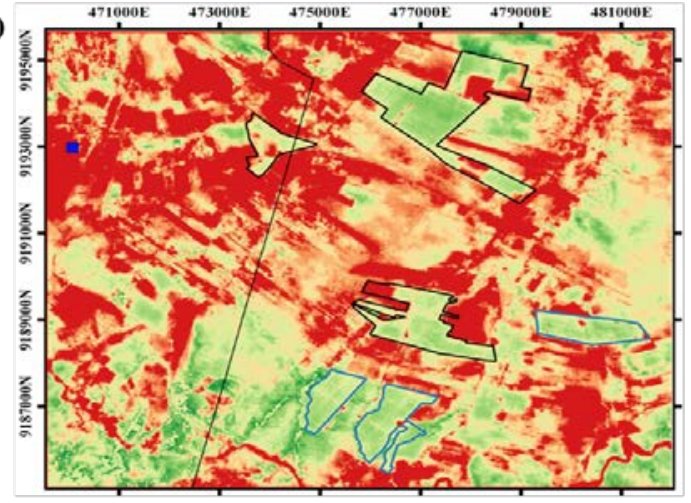

(E)

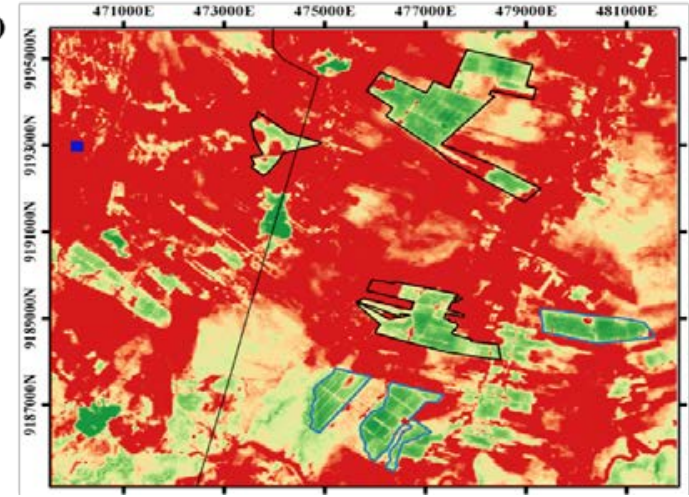

(B)

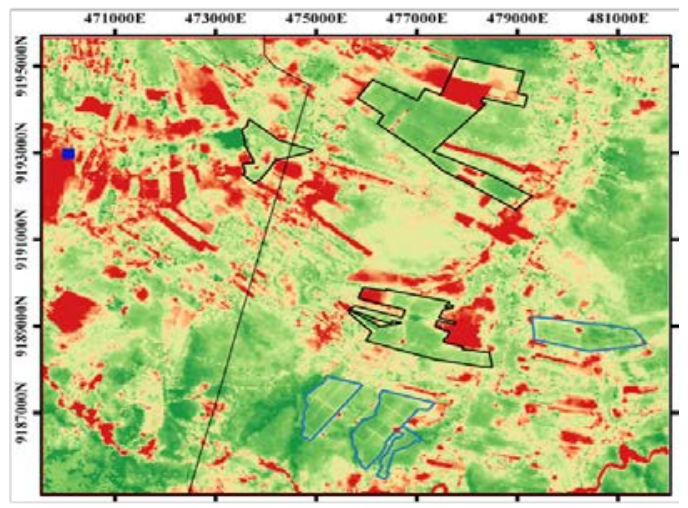

(D)

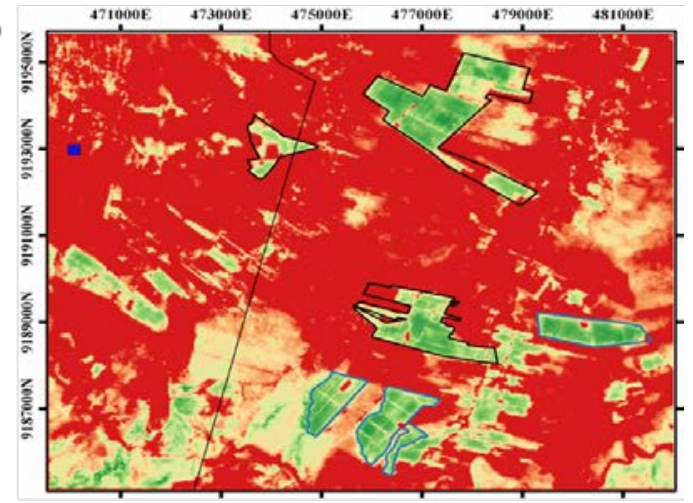

Legenda

$\square$ Limite Municipal

啹rea_Banana_STB

Taxa de evapotranspiração (\%)

50

50
60

$\begin{array}{r}70 \\ 80 \\ \hline 90\end{array}$

$-90$

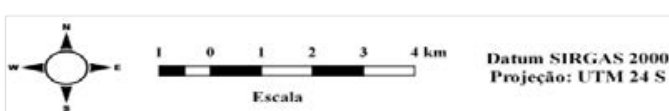

Figura 4. Espacialização dos valores da Taxa de evapotranspiração (\%) da área de estudo no decorrer dos meses: março (A), maio (B), julho (C), outubro (D) e novembro (E) de 2016.

As menores taxas de evapotranspiração foram verificadas nos meses de março e maio, representados nas figuras $4 \mathrm{~A}$ e $4 \mathrm{~B}$, onde é possível observar que estão diretamente correlacionada com o período chuvoso, alta umidade do solo, ocorrência de nebulosidade, presença de cobertura vegetal e temperaturas amenas. No entanto, com o passar dos meses (julho, outubro e novembro) com o início do período de seca, representado nas figuras $4 \mathrm{C}$, 4D e 4E, o déficit pluviométrico contribuiu para a redução da umidade do solo, senescência da vegetação nativa e redução do índice de área foliar deixando o solo descoberto, assim favorecendo o aumento da temperatura da superfície e consequentemente da taxa evaporativa.

A produtividade do cultivo de sequeiro encontrou-se comprometida, uma vez que a inregularidade das chuvas e a umidade do solo baixa, favoreceram a ocorrência de taxas de evapotranpiração entre 50\% e 60\%, indicando portanto que a vegetação encontrase sobre níveis de estrese hídrico extremo. Esse resultado corrobora com Shapland (2015), ao afirmar que taxa de evapotranspiração abaixo de $65 \%$ tende a comprometer o potencial produtivo da cultura que esboça elevada sensibilidade ao déficit hídrico como é o caso da bananeira. 
Os resultados obtidos corroboram com os resultados encontrados por Medeiros et al. (2013), ao afirmam que a localidade de estudo apresenta déficit hídrico na maioria dos meses do ano. Haja vista que os menores valores evaporados ocorrem entre os meses de janeiro a junho e as maiores flutuações entre os meses de julho a dezembro. De acordo com Coelho et al. (2012), em resposta ao déficit hídrico, a bananeira realiza o fechamento dos estômatos, entretanto, este mecanismo de defesa interfere negativamente na assimilação de $\mathrm{CO}_{2}$ e, consequentemente, na produtividade da cultura.

De acordo com o responsável técnico pelo setor de produção da empresa Sítio Barreiras a produtividade média obtida, durante o período de 01 de março a 31 de novembro de 2016, foi de aproximadamente 25,09 ton ha-1 ${ }^{-1}$ valor considerado estável, uma vez que encontra-se inserido no intervalo recomendado por Coelho et al. (2012). O cultivo de banana irrigado da empresa Sítio Barreiras, apresentou taxa evaporativa entre 70 e $100 \%$ durante o período em análise, corroborando com Shapland (2015), ao afirmar que taxas de evapotranspiração entre 75 e $100 \%$ indica que a cultura encontra-se submetida a nível de estresse hídrico baixo, e portanto, o nível de produtividade tende a se manter estável.

A taxa de evapotranspiração observada durante o período em análise da empresa Sítio Paraíso Verde encontrou-se entre 70 e 90\%, em quase totalidade das áreas avaliadas. Entretanto o responsável técnico pelo setor de produção da referida empresa afirma que a produtividade média obtida foi de aproximadamente 35,00 t ha-1. Esse resultado corrobora com Shapland (2015), no entanto, diverge com Coelho et al. (2012).

\section{CONCLUSÃO}

A cultura apresentou taxa evaporativa entre $75 \%$ e $100 \%$ durante o período analisado, logo, esteve submetida a um baixo nível de estresse hídrico; com isso, infere em afirmar que a produtividade situa-se centro do aceitável, visto que o cultivo encontra-se consolidado e estável há mais de dois anos; das duas empresas avaliadas, o Sítio Barreiras foi a que esboçou maior relação eficiência da água/produtividade.

\section{REFERÊNCIAS}

ADECE (Agência de Desenvolvimento do Estado do Ceará). Perfil da Produção de Frutas Brasil e Ceará. In: FRUTAL, 20., 2013, Fortaleza. Palestra... Fortaleza: FRUTAL, 2013. Disponível em:

$<$ http://www.adece.ce.gov.br/phocadownload/ Agronegocio /perfil_da_producão>. Acesso em: 26 nov. 2016.

ALLEN, R. G., TASUMI, M.; TREZZA, R. Satellite-based energy balance for mapping evapotranspiration with internalized calibration (METRIC)Model. Journal of Irrigation and Drainage Engineering, v. 133, p. 380-394, 2007.

ALLEN, R. G.; TASUMI, M.; TREZZA, R.; KJAERSGAARD, J. H. METRIC Mapping evapotranspiration at high resolution. Applications Manual for Landsat Satellite Imagery. Version, v. 2, n. 3, 2005.

ALLEN, R.G. REF-ET: Reference Evapotranspiration Calculation Software. 2002. Disponível em: $<$ http://www.kimberly.uidaho.edu/ref-et/>. Acesso em: 10 dez. 2016.

BRANDÃO, Z. N.; SOUZA, J. Y. B.; BARBOSA, M. P.; ZONTA, J. H.; BEZERRA, J. R. C. Zoneamento de áreas de plantio e mapeamento de APPS e RLS usando imagens ALOS. In: SIMPÓSIO BRASILEIRO DE CIÊNCIAS GEODÉSIAS E TECNOLOGIAS DA GEOINFORMAÇÃO, 4., Recife. Anais... Recife: UFPE, 2012. p. 001-006. 
COELHO, E. F. (Ed.). Irrigação da bananeira. Brasília, DF: EMBRAPA, 2012. 280 p.

FAO (Agricultural Organization of the United Nations). Statistical Databases. Disponível em: <http://www.fao.org>. Acesso em: 24 nov. 2016.

FUNCEME (Fundação Cearense de Meteorologia e Recursos Hídricos). Mapas de levantamento de reconhecimento de média intensidade dos solos da Mesorregião do Sul Cearense, p.98-106. Fortaleza, CE, 2012.

IBGE (Instituto Brasileiro de Geografia e Estatística). Censo agropecuário de 2010. Disponível em: <http://www.ibge.gov.br/cidadesat/topwindo w.htm?1>. Acesso em 24 nov. 2016.

MEDEIROS, R. M.; SILVA, J. A. S.; SILVA, A. O.; MATOS, R. M.; BALBINO, D. P. Balanço hídrico climatológico e classificação climática para a área produtora da banana do município de Barbalha, CE. Revista Brasileira de Agricultura Irrigada, v. 7, n. 4, p. 258 - 268, 2013.

MONTENEGRO, A. A. T.; GOMES, A. R. M.; MIRANDA, F. R.; CRISÓSTOMO, L. A. Evapotranspiração e coeficiente de cultivo da bananeira para a região litorânea do Ceará. Revista Ciência Agronômica, Fortaleza, v. 39, n. 2, p. 203-208, 2009.

ONU (Organização das Nações Unidas). Agricultura é quem mais gasta água no Brasil e no mundo. Disponível em: $<$ http://www.ebc.com.br/noticias/internaciona 1/2013/03/ agricultura-e-quem-mais-gastaagua-no-brasil-e-no-mundo>. Acesso em: 12 dez. 2016.

ROMÁN, M. O.; SCHAAF, C. B.; LEWIS, P.; GAO, F.; ANDERSON, G. P.; PRIVETTE, J. L.; STRAHLER, A. H.
Assessing the coupling between surface albedo derived from MODIS and the fraction of diffuse skylight over spatiallycharacterized landscapes. Remote Sensing of Environment, v. 114, n. 4, p. 738-760, 2010.

SHAPLAND, T. FieldStat: A Superpower for Growers. 2015. Disponível em:< https://www.tuletechnologies.com/blog/fieldst at-a-superpower-for-growers $>$. Acesso em: 24 nov. 2016.

SILVA, B. B. D.; BRAGA, A. C.; BRAGA, C. C.; OLIVEIRA, L. M.; MONTENEGRO, S. M.; BARBOSA JUNIOR, B. Procedures for calculation of the albedo with OLILandsat 8 images: Application to the brazilian Semi-arid. Revista Brasileira de Engenharia Agrícola e Ambiental, v.20, n.1, p.3-8, 2016.

SILVA, B. D.; GALVÍNCIO, J. D.; MONTENEGRO, S. M. G. L.; MACHADO, C. C. C.; OLIVEIRA, L. D.; MOURA, M. D. Determinação por sensoriamento remoto da produtividade primária bruta do perímetro irrigado São Gonçalo-PB. Revista Brasileira de Meteorologia, v. 28, n. 1, p. 57-64, 2013.

SOUZA, J. D.; SILVA, B. B. DA; CEBALLOS, J. C. Surface albedo obtained with MODIS images in cases of low and high aerosol loading in the atmosphere. Revista Brasileira de Geofísica, v.32, p.5-20, 2014.

TEIXEIRA, A. H. de C.; HERNANDEZ, F. B. T.; ANDRADE, R. G.; LEIVAS, J. F.; VICTORIA, D. de C.; BOLF, E. L. Irrigation performance assessments for corn crop with landsat images in the São Paulo state, Brazil. In: INOVAGRI INTERNATIONAL MEETING. 2., 2014, Fortaleza, CE. Anais... Fortaleza: INOVAGRI, 2014. p.739-748.

USGS (United States Geological Survey). Imagens LANDSAT 8 OLI/TIRS. Disponível em: < https://earthexplorer.usgs.gov/>. Acesso em: 20 out. 2016. 